ARSENIC in ANIMALs. - Prof. Ludwig has recently (Wiener Akad. Anz.) inquired into the distribution of arsenic in the animal organism after ingestion of arsenious acid. The objects he examined were the organs of suicides who had poisoned themselves with arsenic, and of dogs which were poisoned, some acutely, some chronically, with arsenic. In all experiments it was found that the arsenic accumulated most in the liver, and that in acute poisoning the kidneys also contained abundant arsenic, whereas in the bones and in the brain there was little of the poison. In case of chronic poisoning with arsenic, where death did not ensue, the poison was found to remain (after ingestion was stopped) longest in the liver, being much sooner excreted from the other organs. The results of this investigation are in direct opposition to those obtained by Scolosuboff, who always found most arsenic in the brain.

DIOPTRICS OF THE EyE.-In the investigation of the dioptric properties of the crystalline lens of the eye, physiologists have hitherto accepted an index of refraction of the lens determined for only one condition of accommodation. It seemed desirable to Herr Matthiessen to attain greater accuracy by ascertaining the dioptric properties of the lens in different states of accommodation, the structure of the lens as now known being fully considered. The subject is discussed at length by him in Pfiuger's Archiv (xix. p. 480). In tabular form he presents a comparison of the positions of the dioptric cardinal points for the human eye and for the eyes of several lower animals, corresponding to different states of accommodation, infinite distance $160 \mathrm{~mm}$. and $100 \mathrm{~mm}$. A com. prehensive list of works on the dioptrics of the lens and the eye generally is added to Herr Matthiessen's paper.

\section{EXPERIMENTAL DETERMINATION OF THE VELOCITY OF LIGHT}

I ET s, Fig 1 , be a slit through which light passes, falling on $\mathrm{R}$, a mirror free to rotate about an axis at right angles to the plane of the paper; $I$, a lens of great focal length, upon which the light falls, which is reflected from $k$. Let $M$ be $a$ plane mirror, whose surface is perpendicular to the line $R$, passing through the centres of $R, I$, and $M$, respectively. If $\mathrm{L}$ be so placed that an image of $\mathrm{S}$ is formed on the surface of $M$, then, this image acting as the object, its image will be formed at $s$, and will coincide point for point with $\mathrm{s}$.

If, now, $R$ be turned about the axis, so long as the light falls on the lens, an image of the slit will still be formed on the surface of the mirror, though on a different part, and as long as the returning light falls on the lens, an image of this image will be formed at $\mathrm{s}$, notwithstanding the change of position of the first image at $M$. This result, namely, the production of a stationary image of an image in motion, is absolutely necessary in this method of experiment. It was first accomplished by Foucault, and in a manner differing apparently but little from the foregoing.

In this case, L, Fig. 2, served simply to form the innage of $S$, at $M$; and $M$, the returning mirror, was spherical, the centre coinciding with the axis of $R$. The lens, $L$, was placed as near as possible to $R$. The light forming the return image lasts, in this case, while the first image is sweeping over the face of the mirror, $M$. Hence the greater the distance, R M, the larger must be the mirror, in order that the same quantity of light may be preserved, and its dimensions would soon become inordinate. The difficulty was partly met by Foucault, by using five concave reflectors instead of one; but even then the greatest distance he found it practicable to use was only twenty meters.

Returning to Fig. I, suppose that $R$ is in the principal focus of the lens, $L$; then if the plane mirror, $M$, have the same diameter as the lens, the first or moving image will remain upon $\mathrm{M}$ as long as the axis of the pencil of light remains on the lens, and this will be the case no matter what the distance may be.

When the rotation of the mirror, $\mathrm{R}$, becomes sufficiently rapid, then the flashes of light which produce the second or stationary image become blended, so that the image appears to be continuous. But now it no longer coincides with the slit, but is deflected in the direction of the rotation, and through twice

${ }^{ \pm}$By Albert A. Michelson, Master, U.S. Navy Read before the American Association. the angular distance described by the mirror, during the time required for light to travel twice the distance between the mirrors. The displacement is measured by its arc, or, rather, by its tangent. To make this as large as possible, the distance between the mirrors, the radius, or distance from the revolving mirror to the slit, and the speed of rotation should be made as great as possible.

The second condition conflicts with the first, for the "radius" is the difference between the distances of principal focus and the conjugate focus for the distant mirror. The greater the "distance," therefore, the smaller will be the "radius." There

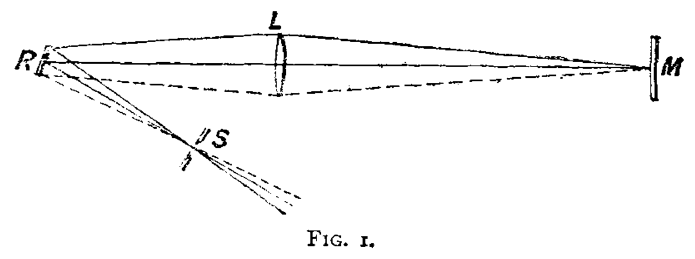

are two ways of solving the difficulty : first, by using a lens of great focal length, and, secondly, by placing the revolving mirror within the principal focus of the lens. Both means were employed. The focal length of the lens was 150 feet, and the mirror was placed about fifteen feet within the principal focus. A limit is soon reached, however, for the quantity of light received diminishes very rapidly as the revolving mirror ap. proaches the lens.

The chief objection urged in reference to the experiments made by Foucault is that the deflection was too small to be measured with the required degree of accuracy. This de-

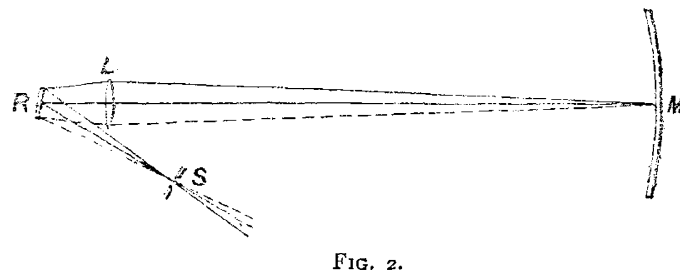

flection was but a fraction of a millimeter, and when it is added that the image is always more or less indistinct on account of atmospheric disturbances, as well as imperfec. tions of lenses and mirrors, it may well be questioned whether the results could be relied upon within less than one per cent.

In the following experiments the distance between the mirrors was nearly 2,000 feet. The radius was about thirty feet, and the speed of the mirror was about 256 revolutions per second. The deflection exceeded 133 millimetres, being about 200 times that obtained by Foncault. If it were necessary it could be

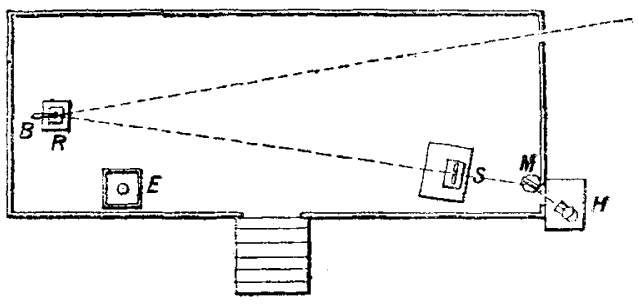

FiG. 3.

still further increased. This deflection was measured within three or four hundredths of a millimeter in each observation. and it is safe to say that the result, so far as it is affected by this measurement, is correct to within one ten-thousandth part.

The site selected for the experiments was a clear, almost level stretch along the north sea-wall of the Naval Academy. A frame building was erected at the western end of the line, a plan of which is represented in Fig. 3

The building was forty-five feet long and fourteen feet wide, and raised so that the line of light was about eleven feet above 
the ground. A heliostat at $\mathrm{H}$ reflected the sun's rays through the slit at $\mathrm{s}$ to the revolving mirror, $\mathrm{R}$, thence through a hole in the shutter, through the lens, and to the distant mirror. The interior of the building was painted black. In a room underneath the part, $R$, was the apparatus which supplied the air for turning the mirror.

The heliostat was kindly furnished by Dr. Woodward, of the Army Medical Museum, and was a modification of Foucault's

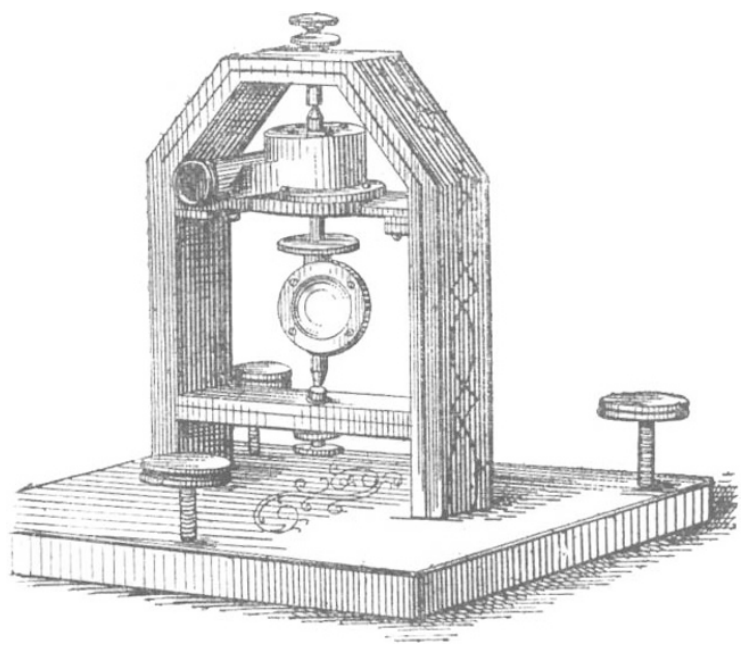

Fic. 4.

form, designed by Keith. It was found to be easy of adjustment and quite accurate.

The light was reffected from the heliostat to a plane mirror, M, Fig. 3 , so that the former need not be disturbed after being ance adjusted.

The revolving mirror was made by Fanth \& Co., of Washington. It consists of a cast-iron frame, Fig. 4, resting on three levelling

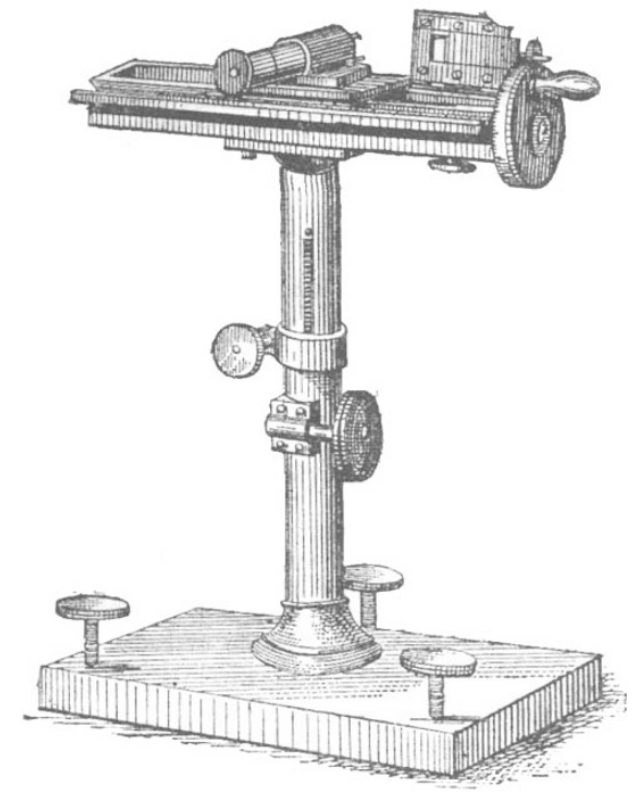

Fig 5 .

screws, one of which was connected by cords to the table at S, Fig. 3, so that the mirror conld be inclined forward or backward while making the observations. Two binding screws, terminating in hardened steel conical sockets, hold the revolving part. This consists of a steel axle, the pivots being hardened, expanding into a ring, which holds the mirror. The latter was a disc of plane glass, made by Alvan
Clark, about one and a quarter inch in diameter and 0.2 inch thick. It was silvered on one side, the reflection taking place from the silvered side. A species of turbine wheel is beld on the axle by friction. This wheel has six openings for the escape of air. The air entering on one side acquires a rotary motion in the box, carrying the wheel with it, and this motion is assisted by the reaction of the air in escaping. The disc above the mirror serves the purpose of bringing the centre of gravity in the axis of rotation. This was done, following Foucault's plan, by allowing the pivots to rest on two inclined planes of glass, allowing the arrangement to come torest, and filing away the lowest pairt of the disc ; then trying again, and so on, till it would rest in indifferent equilibrium. The part corresponding to the disc in Foucault's apparatus was furnished with three vertical screws, by moving which the axis of figure was brought into coincidence with the axis of rotation. This adjustment was very troublesome, and in this apparatus was found unnecessary. When the adjustment is perfect the apparatus revolves without giving any sound, and when this is attained the motion is regular and the speed great. A slight deviation causes a sound, due to the rattling of the pivots in the sockets, the speed is very much diminished, and the pivots commence to wear. In Foucault's apparatus oil was furnished to the pivots, through small holes running through the screws, by pressure of a column of mercury. In this apparatus it was found sufficient, at high speed, to touch the pivots occasionally with a drop of oil.

The quantity of air entering could be regulated by a valve,

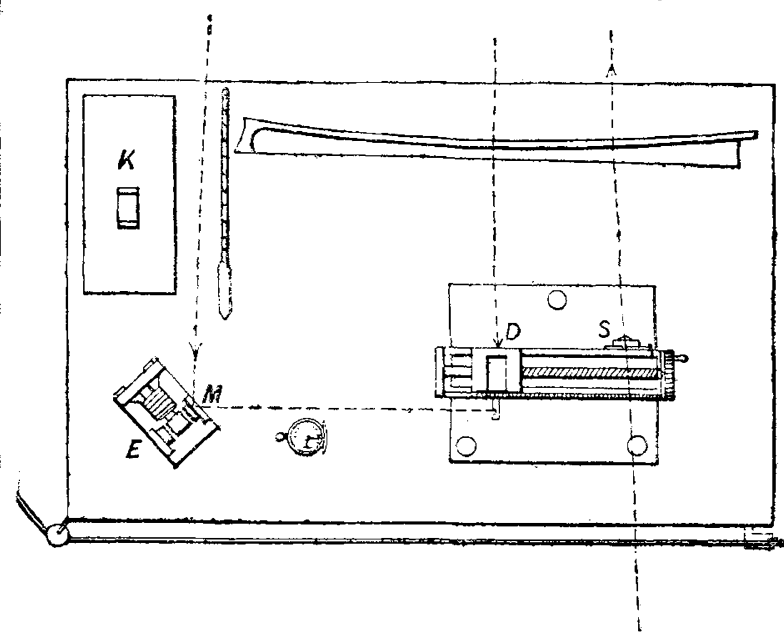

FเG. 6

to which was attached a cord leading to the table. The revolving mirror was mounted on a brick pier

The apparatus for measuring the deflection was made by Grunow, of New York.

It consists of a screw, with divided circle, Fig. 5. To the frame is attached an adjustable slit. On the screw travels a carriage which supports the eyepiece, which consists of an achromatic lens, having in its focus at the end of the tube a single vertical silk fibre.

Next the eye is a piece of plane glass, inclined at an angle of $45^{\circ}$. In measuring the deflection, the eyepiece is moved till the cross hair bisects the slit (with which it is nearly in the same plane), and the reading. of the scale and divided circle gives the position. This is made once for all, unless the slit be altered in width or position. Then the eyepiece is moved till the cross hair bisects the deflected image of the slit; the reading of scale and circle are again taken, and the difference in readings gives the deflection. The screw has no lost motion, so that readings may be taken with the screw turned in either direction. This apparatus is mounted on a standard with rack and pinion, and the base furnished with levelling screws.

To regulate and measure the speed of rotation, a tuning. fork, bearing on one prong a steel mirror, was employed. This was kept in vibration by a current of electricity from five gravity cells. The fork was so placed that the light from the revolving mirror was reflected to the piece of plane glass in the eyepiece, thence reflected into the eye. When fork and revolving mirror are both at rest, the eye sees an image of the revolving mirror. 
When the fork vibrates, this image is drawn out into a band of light. When the mirror commences to revolve, this band breaks up into a number of moving images of the mirror; and when, finally, the mirror makes as many turns as the fork makes vibrations, these images are reduced to one which is stationary. This is also the case when the number of turns is a sub-multiple. When it is a multiple, or a simple ratio, the only difference is that there will be more than one image.

Hence, to make the mirror execute a given number of turns, all that is necessary is to pull the cord attached to the valve, to the right or left, till the images of the revolving mirror come to rest.

The electric fork made about 128 vibrations per second. No dependence was placed upon this rate, however, but at each set of observations it is compared with a standard $\mathrm{Ut}_{3}$ fork, the temperature being noted at the same time. In making the comparison the beats were counted for 60 seconds.

It is interesting to note that the electric fork, as long as it remained untouched and at the same temperature, did not change its rate more than $0^{\circ} 01$ or $0^{\circ} 02$ vibration per second.

Fig. 6 represents the table at which the observer sits. The light from the heliostat passes through the slit at $\mathrm{s}$, goes to the revolving mirror, etc., and on its return forms an image of the slit at $\mathrm{D}$, which is observed through the eyepiece. E represents the electric fork, bearing the steel mirror, $M ; K$, the standard fork on its resonator.

The lens was made by Alvan Clark. It was 8 inches in dia. meter, focal length 150 feet, not achromatic. It was mounted in a wooden frame, placed on a support moving on a slide about 16 feet long, placed about 80 feet from the building. As the diameter of the? lens was so small in comparison with its focal length, its want of achromatism was inappreciable. For the same reason the effect of "parallax" was too small to be noticed.

The stationary mirror was one of those used in taking photographs of the transit of Venus. It was about 7 inches in diameter, mounted in a brass frame capable of adjustment in a vertical and a horizontal plane by screw motion. Being prismatic, it had to be silvered on the front surface. To facilitate adjustment, a small telescope furnished with cross hairs was attached to the mirror by a universal joint. The heavy frame was mounted on a brick pier, and the whole surrounded by a wooden case, to protect it from the sun.

The adjustment was effected as follows :-

A theodolite was placed at about roo feet in front of the mirror, and the latter was moved about, till the observer at the theodolite saw the image of his glass reflected in the centre of the mirror. Then the telescope attached to the mirror was pointed at a mark on a piece of cardboard attached to the theodolite. 'Thus, the axis of the telescope was placed at right angles to the surface of the mirror. The theodolite was then moved to 1, 00 feet, and, if found necessary, the adjustment repeated. Then the mirror was moved till its telescope pointed at the hole in the shutter of the building. The adjustment was completed by moving the mirror by signals, till the observer, looking through the hole in the shutter through a good spyglass, saw the image of the glass reflected centrally in the mirror.

Notwithstanding the wooden case about the pier the mirror would change its position between morning and evening, so that the last adjustment had to be repeated before every series of experiments.

\section{(To be continued.)}

\section{ON THE MOUNTAINS OF THE NORTHERN AND WESTERN FRONTIER OF INDIA}

$T H E R E$ are certain moot questions relating to the mountains of the north-west frontier of India upon which it appears desirable to elicit the opinion of geographers. On this occasion I propose to discuss the western limits of the Himalaya; the northern and southern limits of the Hindu Kush; the parallelism and lateral communications of the ranges between the Hindu Kush and the Aralo-Caspian plain and of other parts of the north-west frontier; and the limits of the Iranian group of highlands, at its junction with the Tibeto-Himalayan and Pamir groups. Finally the proper route of a railway to India between Mesopotamia and the Indus is indicated along a remarkable line of elevated valleys parallel to the coast.

I Paper read at the Sheffield meeting of the British Association by Trelawney W. Saunders.
The Himalaya ranges form a part of the great girdle of mountains which continuously encircle the central portion of the Asiatic Continent, and include the Chinese colonial depend. encies of Ili, Mongolia, Kokonor, and Tibet.

This vast mountain girdle is naturally grouped into four parts corresponding with the outlets of its exterior drainage according to their connection with the Arctic, Pacific, and Indian Oceans, and the Aralo-Caspian Seas respectively. These four divisions of the great mountain girdle have been named from their chief features, (I) the Tibeto-Himalayan, (2) the Yunling, (3) the Altaic, and (4) the Pamir systems. The interlacing or overlapping of these systems at their junctions is not always easy to make out, and presents occasional difficulties like other systems of classification, no matter what the subject may be.

The question of the western termination of the Himalaya relates to two divisions of the great girdle, namely, The TibetoHimalayan and the Pamir, to which must be added another group lying outside those two, but impinging on them at its north-east corner. This group is formed by the Iranian highlands, a compact quadrilateral mass bounded by the lowlands of the.Indus, the Arabian Sea and the Persian Gulf, the lowland of the Tigris and Euphrates, and the Aralo-Caspian plain. The only questions which can well arise with regard to the boundaries of the Iranian mountain system, relate to its north-west and north-east angles, where it unites with the Tauric system on the one hand and the Himalaya and Pamir on the other. It is with the latter anly that we propose to deal now.

My Tibeto-Himalayan system was introduced in 1870 in "A Sketch of the Mountains and River Basins of India in two Maps, with Explanatory Memoirs." It was further developed in the Geographical Magazine, for July, $\mathrm{x} 877$; and I am indebted to the distinguished chairman of this section for an appreciative account of it in two editions of his "Memoirs of the Indian Surveys in 1871 and $1877 . "$ It resolves the leading features of the vast mass of which it treats into four great chains with their outer slopes and intermediate valleys or plateaus. I am obliged to allude briefly to this bygone work, for the purpose of forming a logical basis for the argument which follows.

The northern and southern Himalaya are two of these great chains. The Karakorum-Gangri and the Kuenlun are the other two. The Southern Himalaya rises from the great plain of India, and its culminating summit is distinguished by an extraordinary chain of snowy peaks throughout the whole extent which is claimed for it. The catenary and close succession of these snowy peaks cannot be denied, for they have been fixed in position and altitude by the indubitable observations of the great Trigono. metrical Survey of India. Nor can the existence be disputed of the line of valleys which forms the northern base of this snowy range and distinctly separates it from the Northern Himalaya.

Yet an antiquated theory conceived before the existence of this snowy range was demonstrable, is still held to be possibly tenable by the authors of the recently published manual of the Geology of India, although they do not condescend to any reason for their conclusion.

Now this is not merely a matter of dispute between geologists and geographers, but it is one of the greatest practical importance with reference particularly to the potent question of lateral com. munications about which much has been said lately in reference to the Iranian system in Afghanistan. In the successive valleys following one after the other in the same line, each of which I have specifically named in the Geographical Magazine, there is indisputable evidence of the separation of the two ranges, and of that lateral communication which is an ordinary feature of mountain systems, rather than otherwise.

The Northern Himalaya has its southern base in these valleys, while its northern base is found in the extended trough along which flows the upper courses of the great rivers Indus, Sutlej, and Sanpu.

It is usually said that the Himalaya extends up to the gorge of the Indus on the west and to the gorge of the Sanpu on the east, and this is the extent assigned to the Himalaya by the authors of the "Manual of the Geology of India." But this restriction falls short of the limits which we have already assigned to the TibetoHimalayan system, on the basis of the natural oceanic watersheds. It also falls short of the extension attributed to the Himalaya on the west by observers and geographers of celebrity ; and we shall endeavour to prove that it falls short, on the west at least, of the plain and simple application of the same conditions as those on which the Himalaya is allowed to extend up to the gorge of the Indus. 